\title{
Erratum: Optimized norm-conserving Vanderbilt pseudopotentials [Phys. Rev. B 88, 085117 (2013)]
}

\author{
D. R. Hamann \\ (Received 5 June 2017; published 26 June 2017)
}

DOI: 10.1103/PhysRevB.95.239906

Errors have been found in Eqs. (15) and (16). The correct constraint equation is

$$
x_{1}=s\left[D_{\text {norm }}-\sum_{i=2}^{N-M} x_{i}^{2}\right]^{1 / 2} \text {. }
$$

By inspection of the residual energy $E^{\mathrm{r}}$ given in Eq. (14), it is clear that the sign $s$ of $x_{1}$ at the $E^{\mathrm{r}}$ minimum must be opposite to that of $f_{1}$. Setting the derivatives of $E^{\mathrm{r}}$ with respect to $x_{2}, \ldots, x_{N-M}$ to zero using Eq. (15) for $x_{1}$ yields

$$
x_{i}=-f_{i} /\left(e_{i}-e_{1}-f_{1} / x_{1}\right) .
$$

Since all of the $e_{i}$ 's are positive with $e_{1}$ as the smallest and $f_{1} / x_{1}$ is negative, the denominator in the revised Eq. (16) is always positive. When Eq. (16) is now substituted back into Eq. (15), the magnitude of the right-hand side is seen to be a monotonically decreasing function of $\left|x_{1}\right|$ with maximum value $D_{\text {norm }}^{1 / 2}$ at $x_{1}=0$. Thus a simple interval-halving search on $\left\{0, D_{\text {norm }}^{1 / 2}\right\}$ to find $\left|x_{1}\right|$ satisfying Eq. (15) will yield the unique minimum of $E^{\mathrm{r}}$. The previously described hypersphere search is unnecessary. Whereas the correct form of Eq. (15) was used in the open-source ONCVPSP code, the erroneous version of Eq. (16) was coded and used as originally described. Reference [1] is corrected and employs this simple algorithm.

This error was inconsequential for the accuracy that pseudopotentials generated with this code achieved in representing all-electron atoms. Continuity, generalized norm conservation, and logarithmic derivatives were not compromised. The error resulted in a small sacrifice in convergence optimization since the exact minimum of $E^{\mathrm{r}}$ was not achieved. Extensive testing with the corrected code shows no significant changes in benchmark metrics against all-electron solid-state calculations and nearly undetectable differences in observed convergence. This is attributable to the very large dynamic range spanned by $e_{i}, \sim 10^{6}-10^{8}$, which effectively isolated the damage that could be done by the sign and coefficient errors in Eq. (16). Changes in the published figures in this paper would be imperceptible.

The author thanks M. J. van Setten, M. Giantomassi, and F. Gygi for consultation and testing on the significance of this error.

[1] ONCVPSP, Release 3.3.0, available at www.mat-simresearch.com 